\title{
Researchers at Cuba's National Medical Genetics Center: Pioneering Studies on COVID-19
}

\section{Gail A. Reed MS}

Three fourths of the 175 staff at Cuba's National Medical Genetics Center (CNGM) are women. And women constitute $90 \%$ of the research team working on the Center's largest current projectunlocking the biological secrets of COVID-19 in the Cuban population. They are identifying particularly vulnerable groups and geographies, reviewing therapies applied and long-term sequelae of the disease, and contributing to ongoing vaccine research and trials. Their results are critical to determining effective preventive and treatment strategies as the country moves into the next phases of epidemic control. The national study is the first and only one of its kind in Latin America.

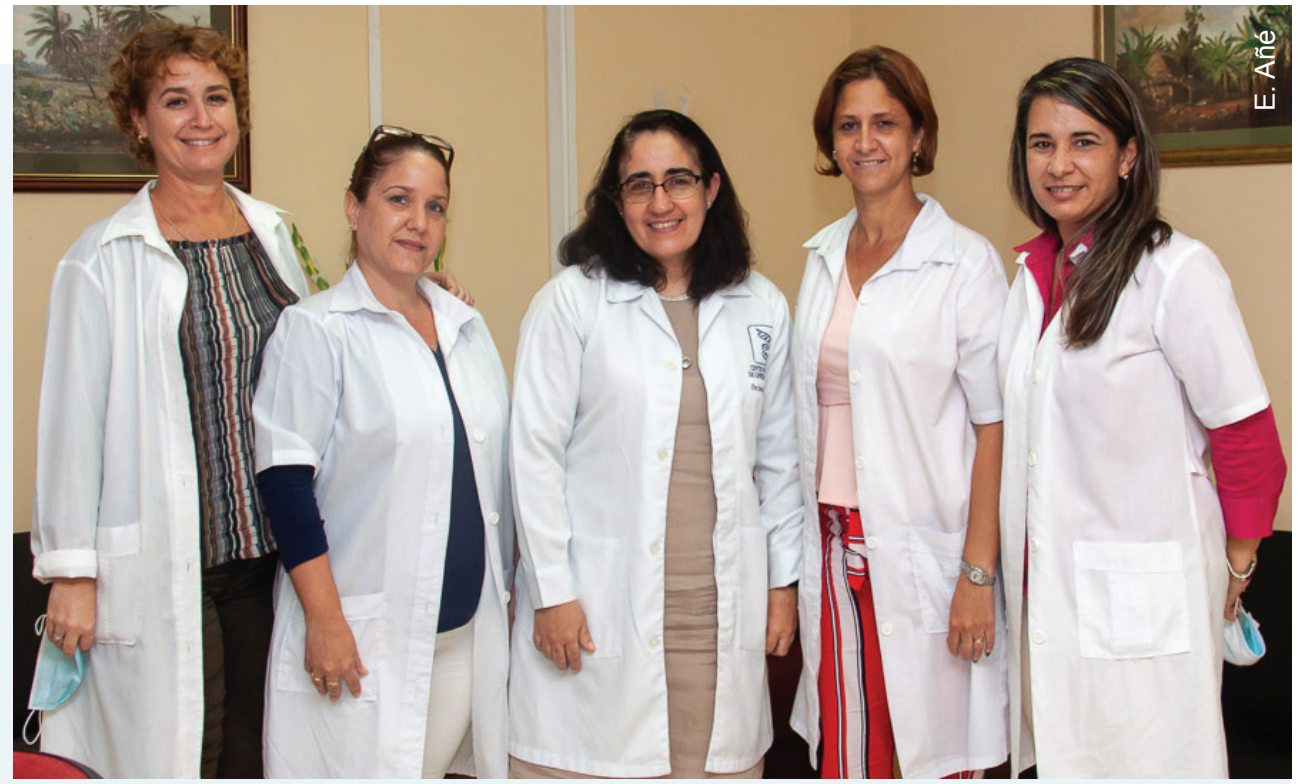

The team (I. to r.): Dr Giselle Monzón, clinical geneticist; Dr Beatriz Suárez, family physician; Dr Beatriz Marcheco, clinical geneticist and CNGM Director; Dr Yaíma Zúñiga, immunologist; and Dr Hilda Roblejo, clinical geneticist.
When the first COVID-19 cases were diagnosed in Cuba on March 11, 2020, the Center's role in epidemiological surveillance was activated, based on its experience with the Zika threat in 2015. This involved mobilizing the National Genetics Network anchored in primary healthcare facilities, comprised of 452 genetics counselors, nurses and clinical geneticists, supported by technicians, epidemiologists and family doctors. The Network's role would become key to the ensuing research.

As the magnitude of the pandemic became clearer, CNGM investigators approached the Ministry of Public Health and government leaders with a broad-ranging proposal to study biological factors that would help explain differences in vulner-

MEDICC Review: Dr Marcheco, can you give us a broad view of what your team is looking for with this national study?

Beatriz Marcheco: The study, although coordinated by our Center, was carried out in primary health care, using all the resources at our disposal from the National Genetics Network. We were already seeing differences in transmission and frequency of the disease in different parts of the country and different population groups. The eastern provinces had fewer cases, for example, while there were more in western Cuba. Why were we seeing such variations? And this was just one of our questions. So we started working with databases from the Ministry of Public Health and genetics departments, then generated guidelines and a participant questionnaire, and began the rollout, which needless to say involved serious logistical planning and attention to PPE and other protection for our team. ability, symptoms, immune response and severity of the disease, as well as its profile in different Cuban subpopulations. After approval, the studies got underway in June, encompassing Cubans who had been infected through June 11, 2020, and were by now convalescing.

The nine main research lines were defined, and principal investigators went to work developing the instruments needed and training personnel across the island on their use. While final results are still being analyzed, CNGM Director Dr Beatriz Marcheco and four lead researchers talked with MEDICC Review about the scope of their work and some of the most intriguing preliminary findings.
The universe of 2233 people who had contracted COVID-19 through June 11 was whittled down to 1182 , our final sample. Eliminated were those who had died, as well as infants under one year old, non-citizens, people who had moved from their area of residence, and 186 who decided not to participate. The final group, from all 15 provinces plus the Isle of Youth Special Municipality, also included health workers.

\section{MEDICC Review: It seems you had a relatively good response. What about those who refused to participate?}

Beatriz Marcheco: We learned a lot about the psychological toll the epidemic has taken on people who contract the disease. Many received us with open arms, anxious that their cases be included. But some just didn't want to hear about COVID-19 again. One man told us that people were closing their doors or crossing the street 
to avoid contact, that he'd become a pariah. Another woman said her downstairs neighbors didn't want her hanging the wash where it might drip on their balcony, for fear of contagion.

This was important to us from a sociological perspective-to see the panic the pandemic had generated. In our meetings with government authorities, we said an effective public messaging campaign was needed, not only to create risk perception, but also to build solidarity... rather than talking about social distancing, to talk about physical distancing and social solidarity. The experience also confirmed our approach: avoiding biological reductionism, and instead taking a comprehensive, integrated look at biological phenomena in social contexts that can either synergize or attenuate their magnitude.

\section{MEDICC Review: Who was involved in the research and how did you proceed?}

Beatriz Marcheco: Some 200 professionals were involved from the National Genetics Network, plus our staff. Upon receiving written informed consent, and before carrying out interviews and clinical exams, our team reviewed all participants' hospital charts. One advantage we had is that all PCR-confirmed cases of COVID-19 in Cuba were hospitalized; none were left at home. So this means researchers could meet with hospital clinicians to get a more precise understanding of individual treatment regimens, since symptoms such as fever could be a result of the disease or secondary to interferon therapy, for example. At the Center, we received the completed interview questionnaires and blood samples for analysis. It was quite a rigorous process, even in the midst of the pandemic.

The study also contemplated a first-degree relative of each patient, one who had cohabitated with the infected person during pre-hospitalization, but who consistently tested negative by PCR. They constituted a control group to compare people who had considerable genetic makeup and living environments in common, but who reacted differently to the virus.

\section{MEDICC Review: What were the main lines of research?}

Beatriz Marcheco: Initially, our priority was an immunogenic analysis of our sample, and we even organized the research at one point around the possibility of carrying out flow cytometry to characterize the cellular subpopulations involved in the body's defenses. Our main aim was a hematological assessment of each individual, including $A B O$ blood group, lymphocyte subpopulations via flow cytometry, and various DNA markers. But flow cytometry requires maintaining the cold chain-impractical given the transport time involved from distant provinces. In the end, we limited this to just under 400 participants.

The main, national study characterized severity of the disease according to several variables. Few investigations have done this, and fewer still have compared convalescing patients to controls who are first-degree relatives. If we look at the lines of research we expect to reflect in scientific papers, I can summarize these, beginning with specific population groups, some of which we can describe in more detail:

- Older adults (persons aged 60 years and older);

- Pregnant women (a case series of 7 women);
- Havana Province, with all the complexities of a national capital; and

- Pediatric patients (137 children, mainly looking at cardiovascular implications, myocarditis in particular).

Then there are the various biological studies, such as:

- The results of flow cytometry, related to lymphocyte subpopulations in both convalescing patients and controls.

- Characterization of molecular markers: tumor necrosis factor alpha (TNF- $\alpha)$, concerning immune response and related symptoms; vitamin D receptor, which plays an essential role in the body's defenses (in particular, we studied genetic characteristics determining ability to retain and process this vitamin); and interleukin 12, which stimulates interferon gamma production. We also studied the ACE2 receptors, the points of cellular entry for the virus (fewer receptors, fewer points of entry).

- Research on symptoms that persisted after initial infection, an investigation carried out in six Havana municipalities.

- Hematological studies involving 31 parameters in over 300 participants, analyses conducted here at the Center, to see how these variables behaved during the convalescent period.

The main study describes results for the whole country, which $\mathrm{Dr}$ Yaíma Zúñiga can explain. It also includes several spinoff investigations: one in cooperation with the Immunoassay Center, another with the Molecular Immunology Center (CIM) and a final one with the Finlay Vaccine Institute (IFV). The Immunoassay Center proposed detection of antibodies specific to SARS-CoV-2. CIM proposed an inhibition assay with samples from the flow cytometry group, to more precisely characterize those antibodies and see to what extent they inhibit coupling of the virus with receptors. IFV proposed a neutralization trial, to study not only inhibition but also whether the antibodies had sufficient 'quality' to neutralize the virus in subsequent exposures. The latter has helped researchers there determine the levels of efficiency required for the Cuban vaccine candidates.

MEDICC Review: Dr Zúñiga, can you introduce yourself and give us an overview of the main national study?

Yaíma Zúñiga: Certainly. I'm an immunologist and have been working here at the Center since 2011. For the main study, our principal aim was to identify immunogenic risk factors related to severity of COVID-19, that is, why some people are asymptomatic while others develop mild or severe forms. One strength of our research was its national character, involving convalescing patients from all 15 provinces and the Isle of Youth Special Municipality, thanks to the National Genetics Network. As Dr Marcheco mentioned, we included as controls first-degree cohabitating family members who remained healthy.

Confirmation of all COVID-19 cases in Cuba is done by PCR. This uniformity of diagnosis constitutes another strength, one not always seen in other studies. And application of a national treatment protocol also made for more standardized therapies.

One challenge was correctly classifying patients according to WHO diagnostic criteria: for example, determining whether those who were asymptomatic at diagnosis actually remained asymptomatic, or whether their diagnosis was simply made in a presymptomatic phase of their illness. This was accomplished by 
NATIONAL MEDICAL GENETICS CENTER STUDIES ON COVID-19 VULNERABILITY, RATES, SYMPTOMS, SEVERE FORMS AND DEATH: CONVALESCING PATIENTS AND CONTROLS, CUBA (MARCH 11, 2020-JUNE 11, 2020)

\begin{tabular}{|c|c|c|c|}
\hline No. & $\begin{array}{l}\text { Study participants/subjects and specific } \\
\text { parameters }\end{array}$ & Number & Comments \\
\hline \multirow[t]{5}{*}{1} & $\begin{array}{l}\text { All convalescing COVID-19 patients in Cuba } \\
\text { ( }>1 \text { year old, Cuban citizen, informed consent } \\
\text { of patient or guardian), plus one first-degree } \\
\text { cohabitating relatives (as controls) }\end{array}$ & $\begin{array}{l}\text { Patients: } 1182 \\
\text { Controls: } 500 \\
\text { Total: } 1682 \\
\text { Plus: } 84 \text { deceased }\end{array}$ & $\begin{array}{l}\text { Carried out in all } 15 \text { Cuban provinces and the Isle of } \\
\text { Youth Special Municipality; includes } \pm 200 \text { health workers; } \\
\text { demographic and biological characteristics related to risk of } \\
\text { disease onset, symptoms, severity and death }\end{array}$ \\
\hline & Persistent symptoms & 67 & $\begin{array}{l}\text { Convalescent patients studied in six municipalities of } \\
\text { Havana Province }\end{array}$ \\
\hline & Hematological profile & 344 & $\begin{array}{l}\text { Includes } 31 \text { parameters (complete blood count, ABO blood } \\
\text { group, etc.) }\end{array}$ \\
\hline & Molecular markers & $\begin{array}{l}\text { TNF- } \alpha: 1028 \\
\text { Vitamin D receptor: } 133 \\
\text { ILK12: } 128 \\
\text { ACE2 receptor gene: } 400\end{array}$ & $\begin{array}{l}\text { Tumor necrosis factor alpha (TNF- } \alpha \text { ); vitamin D receptor; } \\
\text { interleukin 12, ILK12 (stimulates interferon gamma produc- } \\
\text { tion); and ACE2 receptor gene }\end{array}$ \\
\hline & Immunological status via flow cytometry & 389 & \\
\hline 2 & Subpopulation: older adults ( $\geq 60$ years old) & Living: 241 & $\begin{array}{l}\text { Patients from all except Granma Province; includes } 14 \\
\text { health workers }\end{array}$ \\
\hline 3 & Subpopulation: pregnant women & 7 & Extended to their newborns \\
\hline 4 & Subpopulation: Havana Province & 431 & $\begin{array}{l}\text { All } 15 \text { municipalities (In Cuba, Havana Province is also the } \\
\text { capital city.) }\end{array}$ \\
\hline 5 & Subpopulation: pediatric patients & 137 & Emphasis on cardiovascular sequelae, myocarditis \\
\hline
\end{tabular}

NOTES: All confirmed COVID-19 patients in Cuba are hospitalized. Convalescing patients included in the study were those who were RT-PCRpositive from March 11, 2020 through June 11, 2020, and had received at least two negative PCRs (first at hospital and second 15 days after discharge). Controls were RT-PCR-negative fist-degree relatives cohabitating with sick individuals during the pre-hospitalization phase of infection. Postmortem analysis included 84 deceased from COVID-19, for whom demographics (age, sex, etc.) and comorbidities were considered.

reviewing patients' hospital charts and through interviews. Interestingly, although $60 \%$ to $70 \%$ had originally been classified as asymptomatic, this portion dropped considerably through reclassification, since they developed symptoms later. We also found that adults fell ill more often than children, but that children were more often asymptomatic, making them silent carriers.

As far as genetic factors, we analyzed such things as skin color, which is difficult in Cuba where there is so much mestizaje, which means less relation between skin color and actual genetic ancestry. Some US and European literature had reported higher rates as well as greater severity and higher mortality among minorities-Black, Asian, Hispanic. However, we found that disease distribution mirrored fairly well the proportion of people of different skin colors in the Cuban population as a whole (according to census data). We did find differences when it came to severity and mortality, which we are still analyzing. It may be that being mestizo is a protective factor.

\section{MEDICC Review: Have you accounted for other confound- ing variables, having to do with environment, socioeconomic status, etc.?}

Beatriz Marcheco: We didn't carry out a detailed sociological study. We did include employment, educational level and where participants lived-whether in urban or rural areas. Once a person becomes sick, various factors begin to influence outcomes: age, comorbidities, blood group and also skin color. Who are the people who have become seriously ill, at least in Cuba? Most often, these are older people. Which leads us to a number of

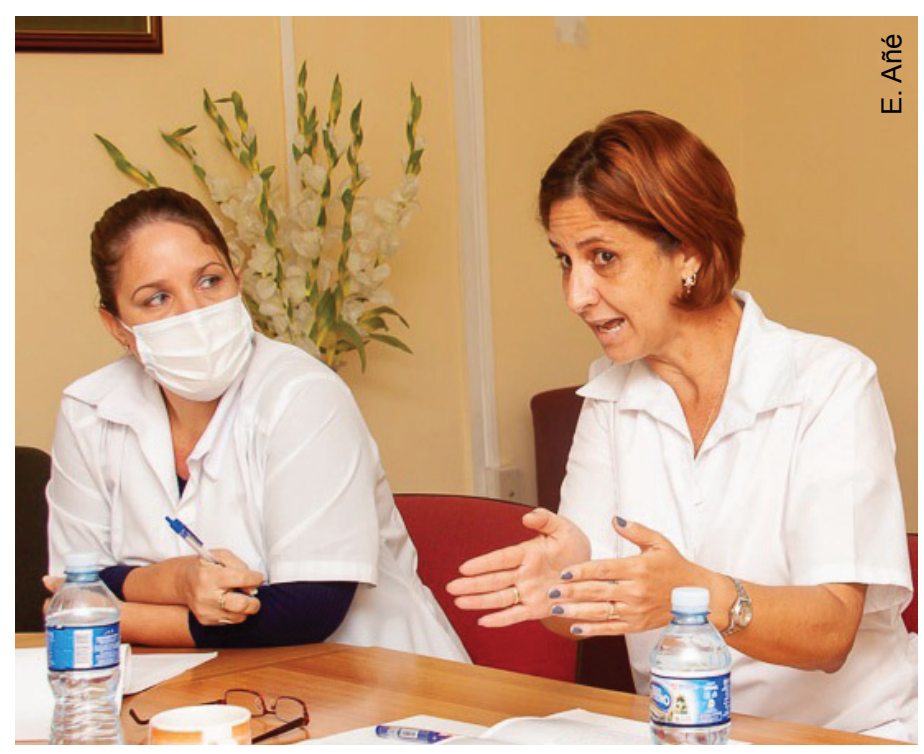

"One strength of the study was its national character, involving convalescing patients from all 15 provinces," Dr. Yaíma Zúñiga.

social and historical variables. In the case of skin color, you would have to look into the nutritional levels and socioeconomic conditions that prevailed for darker-skinned people some 60 years ago, when they were growing up and the basis of their health status was being determined. Those conditions were certainly more precarious than for lighter-skinned people. No matter that Cuba has moved the needle towards equity, that past is important. 
Yaíma Zúñiga: We confirmed what other international studies are showing: that advanced age is indeed a risk factor for developing severe COVID-19 and for death. Our aim was to quantify this risk by comparing different age groups, as ages climbed. The educational level in our study was high, with more university graduates than in the general population. The fact that some 200 health workers, primarily doctors and nurses, were included may have influenced this (thankfully, with no deaths in that subpopulation).

We also looked at blood types. We didn't find any important association of infection risk with Rh factor. However, when we analyzed ABO blood groups, we found persons in blood group A were more apt to progress to severe forms of the disease. Concerning comorbidities in the Cuban population, it is clear that diabetes mellitus and cancer present the most risk, particularly for severe COVID-19 and death. And our results coincided with others that indicate greater risk of death for men.

\section{MEDICC Review: You also analyzed post-mortem cases?}

Yaíma Zúñiga: Yes, 84, determined by death certificates, the total number of deaths from COVID-19 during the period. We analyzed them on the basis of data from the Ministry of Public Health's Statistics Division and the records of the national group that monitors serious and critical cases. For patients who survived, we also looked at antibody response to SARS-CoV-2: in particular, the quantity and quality of antibodies during convalescence, important for predicting re-infection. And also if these antibodies are capable of neutralizing the virus if a person is re-exposed. This is still a big question. Our convalescents were for the most part PCR negative for at least 60 days when studied, and what we began to see was a relation between antibody response and disease severity: the more severe the form, the greater the antibody response. As Dr Marcheco mentioned, this is important for determining what characteristics a vaccine must have to be effective.

\section{MEDICC Review: Turning to Havana, where over two million Cubans live, and which has been the epicenter of the island's COVID-19 epidemic...Dr Roblejo?}

Hilda Roblejo: I've been a clinical geneticist here at the Center since 2009. The cases we studied here in Havana were more than a third of all those in our sample, 431 convalescing patients. Like all capitals, Havana presented its own complexities. The disease was disseminated in all municipalities, unlike some other provinces. So our research team interviewed people in virtually all neighborhoods.

Many aspects of the Havana sample mirrored that of the country as a whole. Most of our patients did develop symptoms; the average age was in the 40s (nationally it was 45 years old). An earlier study looked at advancing age as a risk factor, and identified Plaza as a high-risk municipality, since it is the 'oldest' in the country. On the map, Plaza, Playa, Central Havana...these areas contain some of the most aged populations in the country. In Central Havana, you also have the added risk factor of overcrowding, and the same goes for 10 de Octubre Municipality, with high population density.

In terms of blood group, our results were slightly different from the national findings: we found blood group $\mathrm{O}$ to represent a greater protective factor than we saw nationally.
MEDICC Review: In addition to blood group, were there any other findings that separated Havana from the rest of the sample?

Hilda Roblejo: We found that being asthmatic was actually a protective factor against the severe forms of COVID-19. We think asthmatics fared better due to the therapies most often prescribed, which include immunomodulators, medications that modulate ACE2 receptor production.

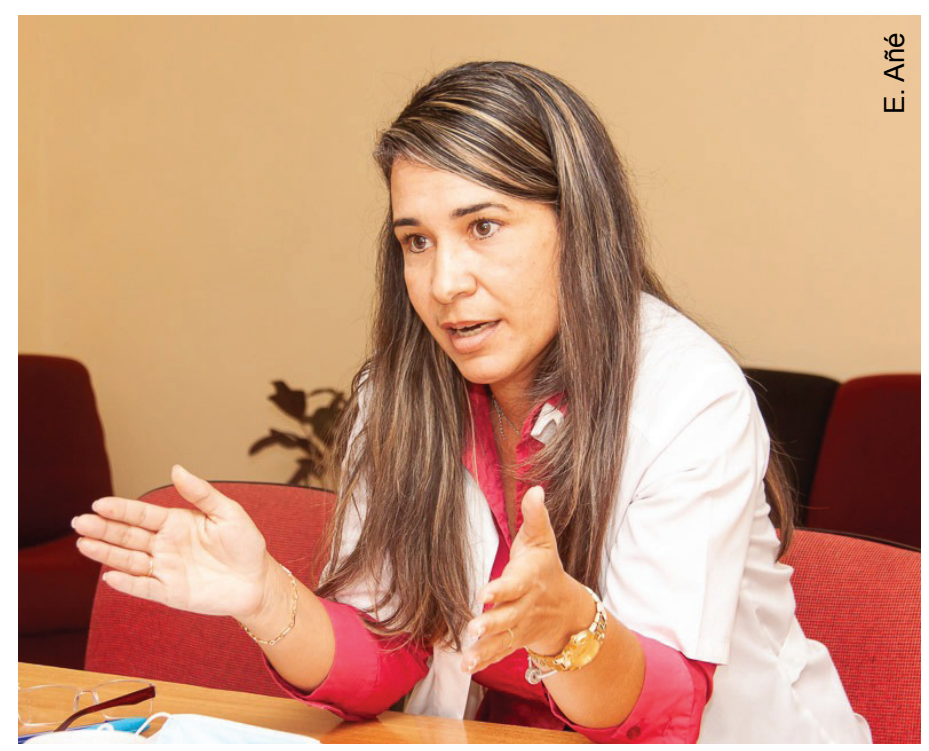

In Havana, "we found that being asthmatic was actually a protective factor against the severe forms of COVID-19," Dr Hilda Roblejo.

MEDICC Review: Dr Suárez, can you introduce yourself and talk about the research facets that concentrated on older convalescent patients?

Beatriz Suárez: I'm a specialist in family medicine with a master's degree in medical genetics. I've worked here at the Center since 2009. My main responsibility is to coordinate the services of the National Genetics Network across the country.

In Latin America, Cuba's is one of the most rapidly aging populations. So for this part of the study, we concentrated on persons 60 years and older, which corresponded to $22 \%$ of those infected during the study period, a good share of whom had either developed severe forms or died from the disease.

The highest concentrations of convalescing patients and also older patients were in Villa Clara, Havana and Matanzas Provinces. Villa Clara, the 'oldest' province, also experienced an outbreak at a nursing home. In Matanzas, cases were concentrated in Cárdenas Municipality, close to the resort town of Varadero, which made it particularly vulnerable given that the first COVID-19 wave in Cuba was determined mainly by imported cases. And Dr Roblejo already spoke about Havana's characteristics.

We studied 241 patients in all, and found slight increases in severe forms according to advancing age. Fourteen of these patients were health workers, and although most had already retired, $22 \%$ were still working. 
As in the international literature, we saw greater risk of infection as the number of comorbidities increased. Among the comorbidities, hypertension was the most frequent, found in half these older adults. Most of these patients contracted COVID-19 with mild or moderate symptoms, and we described the more common ones.

\section{MEDICC Review: What are some of the most important risk factors leading to death?}

Yaíma Zúñiga: More men die than women. Worldwide, we see that men have more risk of developing severe forms of COVID-19 and of dying. We didn't find this correlation with severity of disease in Cuba. However, Cuban men were more at risk of dying. And another interesting thing is that the women who died were older than the men who died. We know that women in general have a better immune response, due to various factors. As an aside, I should also note that more women agreed to participate in our study than men...something we've experienced in other research.

\section{MEDICC Review: What did you find with the group of wom- en who were pregnant when they contracted COVID-19? Dr Monzón?}

Giselle Monzón: I'm originally from Camagüey Province, but have been at the Center since 1998, when I specialized in medical genetics here.

Pregnant women have constituted a vulnerable group in past respiratory disease epidemics, where we have seen them at greater risk of developing serious complications requiring ICU care, and of dying. So this is of grave concern to us as we confront COVID-19.

Through September 2020, PAHO reported over 60,000 pregnant women infected in 14 countries of the Americas, with a mortality of $1 \%$. The highest mortality was reported in Mexico, Brazil and the United States. In Cuba through July 31, 2020, 23 cases had been confirmed among pregnant women, including 16 during the period of our study. Seven women participated in our research, about $44 \%$ of the total sample. In their case, our aim was to identify genetic risk factors associated with more serious disease.

In addition to the interview, we were able to follow the outcome of each pregnancy. All this was possible thanks to the National Genetics Network and the National Maternal-Child Program. This program, based in primary health care, leads to early monitoring of pregnant women in medical services, well before their third trimester, different from what happens in many other countries. And COVID-19 cases in this population were also identified mainly in the community, as a result of active case finding and contact tracing. This enabled early diagnosis by PCR and immediate hospitalization. Pregnant women, according to our national protocols, received antiviral therapy, including interferon alfa- $2 b$, which appears to help explain why none developed serious complications or died.

They did develop mild to moderate symptoms. Interestingly, fever was not one of the main ones most frequently described.

While this was a small sample, we didn't see secondary effects of medications reported by some other patients, either for their own health or for the healthy development of the fetus or newborn. We also carried out a genetic assessment of the neonates.

MEDICC Review: Do you have any evidence of vertical transmission of the SARS-CoV-2 virus?

Giselle Monzón: No, no evidence of vertical transmission, either in the study, or in Cuba up to now. Any transmission has been associated with inadequate management via maternal aerosols and so on.

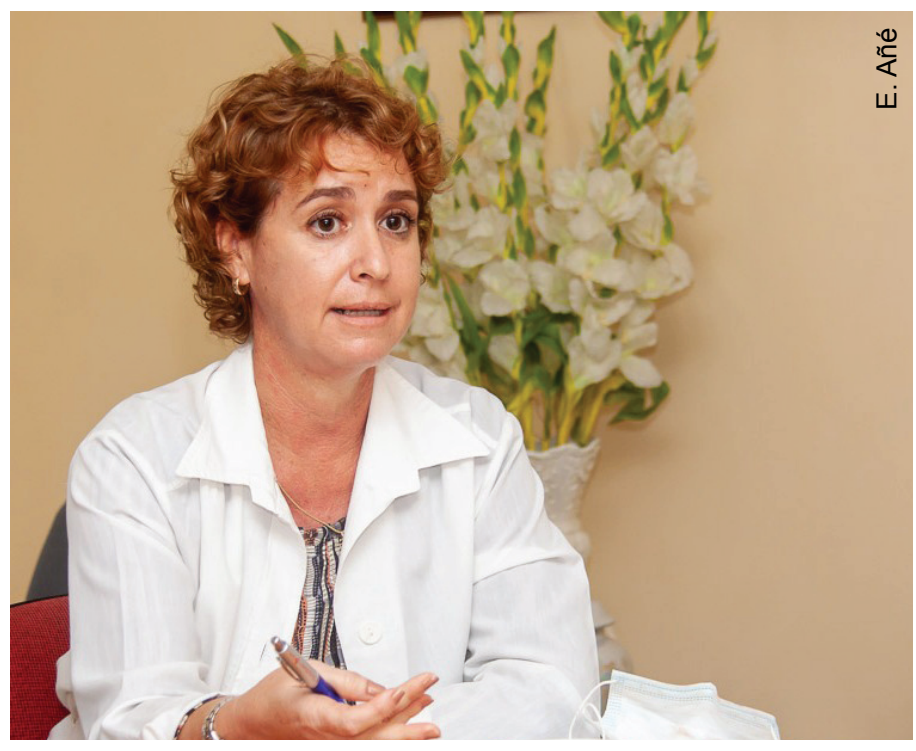

"No evidence of vertical transmission, either in the study, or in Cuba up to now," Dr Giselle Monzón.

MEDICC Review: What are the implications of your findings thus far for Cuban treatment protocols and for the vaccines being developed by Cuban biotech?

Yaíma Zúñiga: In terms of the vaccines, we are determining the antibody titers and the capacity of these antibodies to neutralize the virus, that is, what level of immunogenicity is required-establishing that threshold. To date, the FDA has said that titers must be equal or greater than $1 / 160$ to guarantee neutralization, the level considered by the current vaccine candidates. But when you design a vaccine, you have a given population as a reference, since populations may respond differently. So studying this in our own population is important, a first approximation.

Hilda Roblejo: Our research didn't go as far as to include pharmacogenetic markers that would permit analysis of interferons and other therapies in our treatment protocol, to determine a relation between patient response and these markers. But we have stored DNA samples of all participants for further study of these aspects, especially because this will help design more personalized treatments.

Thus far, what we have been able to do is stratify risk and vulnerabilities, and as a function of this, personalize interventions. On this basis, once Cuba has a vaccine, we can make recommendations on who should be vaccinated first....groups such as older adults, health workers in Havana, people in blood group A, and so forth. 
Beatriz Marcheco: That's what I would emphasize: this research contributes results to the health system for decision-making on priority actions, based on a better stratification of vulnerabilities.

Yaíma Zúñiga: Not only therapeutic management but also for preventive approaches, what to do epidemiologically, what kinds of measures to adopt for different populations. Because I think a strength we have is that we now have DNA and sera from these patients, representing the first group in Cuba that was infected with SARS-CoV-2.

\section{MEDICC Review: We're seeing new variants of the virus emerge through mutations.}

Beatriz Marcheco: The Pedro Kourí Tropical Medicine Institute (IPK) is sequencing genes of cases that have entered from countries in Europe where one new variant has been reported, to see if it is circulating in the country. I expect so. They are also sequencing variants from outbreaks in Ciego de Ávila Province, to see if they are different from others circulating in Cuba. In the case of Ciego de Ávila, this is an important question, since the frequency of cases there, the transmissibility, severity and mortality have been greater than in other regions of Cuba.

We have some observations...we know, for example, that there have been more asymptomatic cases in Ciego de Ávila than elsewhere, which could facilitate greater transmission. And there are genetic markers that also indicate different disease behavior in

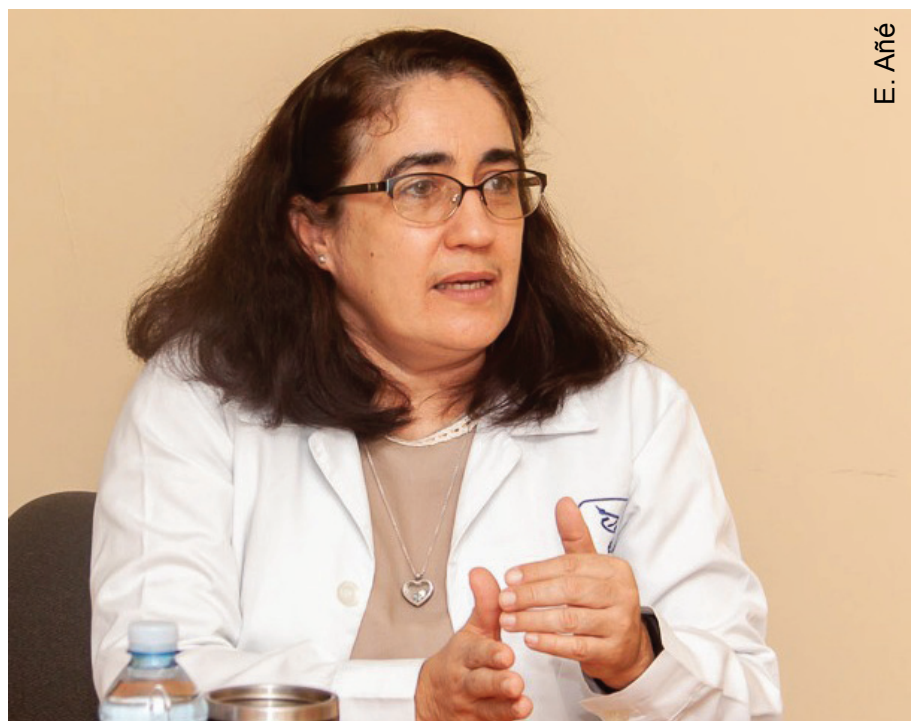

"...the most far-reaching population-based study (on COVID-19) reported in Latin America," Dr Beatriz Marcheco. that province: antibody levels are lower, protection against new exposure is less, and so on.

MEDICC Review: Is that due to the patients themselves or to a different viral strain?

Beatriz Marcheco: That's the question. We don't know. That's what we need to find out.

MEDICC Review: And there is speculation on whether the vaccines being developed will be effective against new mutations.

Beatriz Marcheco: And that's my question, too.

Yaíma Zúñiga: Generally, vaccine designs take into account the immunogen capable of the best neutralizing response. As long as the changes implied by mutations don't involve that specific immunogen, then there should be no problem. But that's the key factor.

MEDICC Review: Returning to your research: are there findings that are particularly important or novel for Cuba or for the world?

Beatriz Marcheco: I think this is the most far-ranging populationbased study that has been reported in Latin America, contemplating the entire country. In terms of particular aspects: we described distribution of symptoms in children and adults, the patterns of symptom presentation, and the relation of these to the risk of developing severe forms of the disease, which we haven't seen in other research.

Characterization of blood groups in such a large number of convalescing patients has only been published in Spain and Italy, not for Latin American populations where the frequency composition of blood groups is different. In Cuba, it's even different by provinces, depending on genetic ancestry. That is, those with more African genetic ancestry are more frequently blood group $\mathrm{O}$, which in some populations appears to be a protective factor against infection, although not against developing severe forms.

And the other important thing is that all these findings contribute to a national strategy for confronting COVID-19, particularly ongoing prevention efforts. Because we now have results that not only consider research from other countries, but most importantly detail quite precisely how the disease is behaving right here in our own population. And thankfully, through the Ministry of Public Health and the experts working on COVID-19, we have a direct line to the decision-makers. $-1 /$ - 